\title{
Interactions among genes in the ErbB-Neuregulin signalling network are associated with increased susceptibility to schizophrenia
}

\author{
Isabel Benzel*1, Aruna Bansal2, Brian L Browning3,7, Nicholas W Galwey², \\ Peter R Maycox ${ }^{1}$, Ralph McGinnis ${ }^{2,8}$, Devi Smart ${ }^{2}$, David St Clair ${ }^{4}$, \\ Phillip Yates ${ }^{5}$ and Ian Purvis 6
}

\begin{abstract}
Address: ${ }^{1}$ Psychiatry CEDD, GlaxoSmithKline, New Frontiers Science Park, Third Avenue, Harlow, Essex, CM19 5AW Harlow, Essex, UK, ${ }^{2}$ Discovery and Pipeline Genetics, GlaxoSmithKline, Harlow, Essex, UK, ${ }^{3}$ Discovery and Pipeline Genetics, GlaxoSmithKline, Research Triangle Park, North Carolina, USA, ${ }^{4}$ Department of Mental Health, University of Aberdeen, Institute of Medical Sciences, Aberdeen AB25 2ZD, UK, ${ }^{5}$ Scottish National Blood Transfusion Service, Aberdeen AB25 2ZW, UK, ${ }^{6}$ Therapeutic Area Team, GlaxoSmithKline, Stevenage, Hertfordshire, UK, ${ }^{7}$ Department of Nutrition, The University of Auckland, Private Bag 92019, Auckland, New Zealand and ${ }^{8}$ Wellcome Trust Sanger Institute, Cambridgeshire, CB10 1SA, UK
\end{abstract}

Email: Isabel Benzel* - Isabel.M.Benzel@gsk.com; Aruna Bansal - Aruna.T.Bansal@gsk.com; Brian L Browning - b.browning@auckland.ac.nz; Nicholas W Galwey - Nicholas.W.Galwey@gsk.com; Peter R Maycox - Peter.R.Maycox@gsk.com; Ralph McGinnis - rm2@sanger.ac.uk; Devi Smart - Devi.Smart@hotmail.co.uk; David St Clair - dl.stclair@abdn.ac.uk; Phillip Yates - philip.yates@nhs.net;

Ian Purvis - i.purvis3@ntlworld.com

* Corresponding author

Published: 28 June 2007

Behavioral and Brain Functions 2007, 3:31 doi:10.1186/1744-908I-3-31
Received: 18 May 2007

Accepted: 28 June 2007

This article is available from: http://www.behavioralandbrainfunctions.com/content/3/I/3 I

(C) 2007 Benzel et al; licensee BioMed Central Ltd.

This is an Open Access article distributed under the terms of the Creative Commons Attribution License (http://creativecommons.org/licenses/by/2.0), which permits unrestricted use, distribution, and reproduction in any medium, provided the original work is properly cited.

\begin{abstract}
Background: Evidence of genetic association between the NRGI (Neuregulin-I) gene and schizophrenia is now well-documented. Furthermore, several recent reports suggest association between schizophrenia and singlenucleotide polymorphisms (SNPs) in ERBB4, one of the receptors for Neuregulin-I. In this study, we have extended the previously published associations by investigating the involvement of all eight genes from the ERBB and NRG families for association with schizophrenia.
\end{abstract}

Methods: Eight genes from the ERBB and NRG families were tested for association to schizophrenia using a collection of 396 cases and I,342 blood bank controls ascertained from Aberdeen, UK. A total of 365 SNPs were tested. Association testing of both alleles and genotypes was carried out using the fast Fisher's Exact Test (FET). To understand better the nature of the associations, all pairs of SNPs separated by $\geq 0.5 \mathrm{cM}$ with at least nominal evidence of association $(P<0.10)$ were tested for evidence of pairwise interaction by logistic regression analysis.

Results: 42 out of 365 tested SNPs in the eight genes from the ERBB and NRG gene families were significantly associated with schizophrenia $(P<0.05)$. Associated SNPs were located in ERBB4 and NRGI, confirming earlier reports. However, novel associations were also seen in NRG2, NRG3 and EGFR. In pairwise interaction tests, clear evidence of gene-gene interaction was detected for NRGI-NRG2, NRGI-NRG3 and EGFR-NRG2, and suggestive evidence was also seen for ERBB4-NRGI, ERBB4-NRG2, ERBB4-NRG3 and ERBB4-ERBB2. Evidence of intragenic interaction was seen for SNPs in ERBB4.

Conclusion: These new findings suggest that observed associations between NRGI and schizophrenia may be mediated through functional interaction not just with ERBB4, but with other members of the NRG and ERBB families. There is evidence that genetic interaction among these loci may increase susceptibility to schizophrenia. 


\section{Background}

Schizophrenia is a complex psychiatric disorder which affects $0.5-1 \%$ of the world wide adult population. A number of putative schizophrenia susceptibility genes have been identified recently [1,2]. Genomewide linkage analysis on large Icelandic pedigrees showed evidence for linkage with an initial LOD score of 3 on chromosome 8p13, which led to the identification of the Neuregulin-1 gene (NRG1) as a potential genetic risk factor (O.R. = 2) for schizophrenia [3]. The association was confirmed in a large Scottish case control data set [4], and both studies indicated a core haplotype $\left(\mathrm{HAP}_{\mathrm{ICE}}\right)$ as the DNA variation carrying most of the risk for schizophrenia. Subsequently, 15 published studies in schizophrenia data sets of various Asian and Caucasian ethnic backgrounds have detected association in NRG1 SNPs or haplotypes, while only four studies were not able to replicate the association (see [5] and [6] for a comprehensive review). The association studies reported thus far strongly suggest a true effect of NRG1 as a susceptibility gene for schizophrenia. However, some caution should be exercised before calling NRG1 a true "schizophrenia gene" due to the lack of a known functional effect of the identified NRG1 variants, the allelic heterogeneity reported across several studies and the multiple SNPs and haplotypes analysed in different studies. Nonetheless, several recent publications report an association between schizophrenia and ErbB4, one of the receptors for NRG1 [7-9]. This provides indirect evidence supporting NRG1 association with schizophrenia and a role for aberrant neuregulin signalling in this disorder.

The Neuregulin-ErbB signalling network is involved in a multitude of processes in the developing and adult brain. Neuregulins for example promote neuronal migration and differentiation, regulate the expression of neurotransmitter receptors, influence glial proliferation, survival and differentiation and play a role in synaptic plasticity.

The neuregulins (NRG) are cell-cell signalling proteins that are ligands for receptor tyrosine kinases of the ErbB family. Whereas NRG1 is known to play essential roles in nervous system and heart development, as well as in the adult brain (see above), less is known about the other members of the NRG gene family, NRG2, -3 and -4 $[10,11]$.

The ErbB family comprises four homologous typeI receptor tyrosine kinases (RTKs). The EGFR (epidermal growth factor receptor, HUGO name for ErbB1), ErbB3 and ErbB4 receptors can bind ligands, whereas ErbB2 lacks a ligand binding domain and functions as a preferred and very potent co-receptor. ErbB3 is devoid of an active kinase domain. ErbB4 is the only ErbB family member that binds all four neuregulins, as well as several proteins that had originally been identified as EGFR ligands (Fig. 1). Furthermore, NRG1 and NRG2 can also bind to ErbB3. In contrast, none of the neuregulins bind to EGFR which instead binds unrelated ligands such as EGF, amphiregulin and others (see Fig. 1).

Ligand binding to the extracellular domain of ErbB family members leads to receptor homo-and heterodimerisation and activation of various intracellular signalling pathways such as the Ras-Raf-MAPK and the PI-3 Kinase pathways (reviewed in [12]).

In order to investigate the involvement of neuregulin pathway genes in schizophrenia, beyond the previously published association with NRG1 and ERBB4, we have tested all eight genes from the ERBB and NRG families for association with schizophrenia. We have investigated both single genes and gene-gene interactions, using a collection of schizophrenia cases and blood bank controls from Aberdeen, Scotland.

\section{Methods \\ Subjects}

396 Caucasian cases were ascertained in Aberdeen, Scotland. All have a basic diagnosis of schizophrenia or schizoaffective disorder according to the Operational Cri-

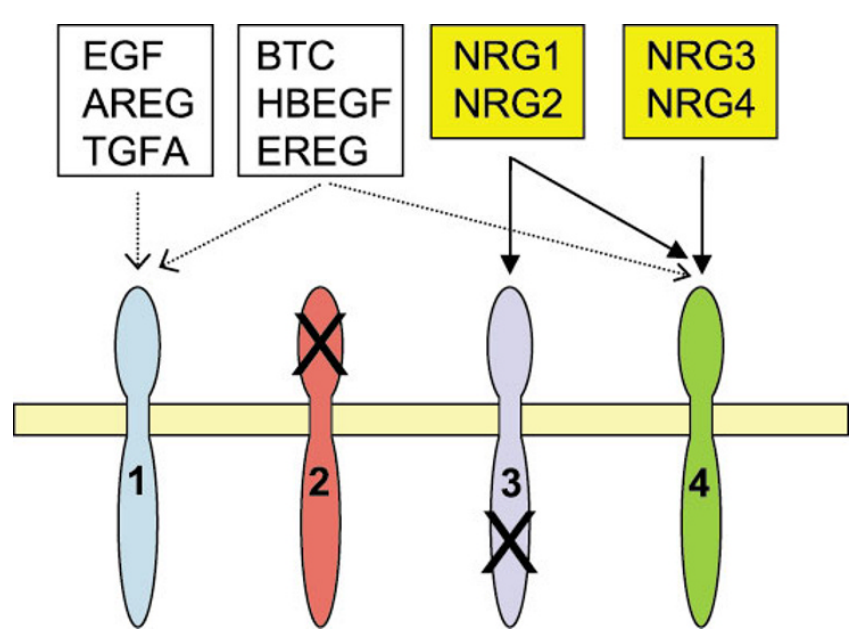

Figure I

Binding specificities of ErbB receptor family ligands. EGF (epidermal growth factor), AREG (amphiregulin) and TGFA (transforming growth factor- $\alpha$ ) bind EGFR (ErbBI) only. BTC (Betacellulin), HBEGF (heparin-binding EGF-like growth factor) and EREG (Epiregulin) bind EGFR and ErbB4. NRGI and NRG2 bind ErbB3 and ErbB4. NRG3 and NRG4 are specific for ErbB4. Receptors form homo- and heterodimers after ligand-binding (not shown). As indicated $(X)$, ErbB2 has no ligand-binding capacity and ErbB3 has no active kinase domain. Only NRG I-4 and ERBBI-4 genes were investigated in this study. (Modified from [46]). 
teria Checklist (OPKRIT). 285 of the cases were male; 111 were female. The mean recruitment age was $44 \mathrm{yr}$ with a standard deviation of $14 \mathrm{yr}$.

1342 Controls were ascertained as a series of anonymous blood donors from Aberdeen, Scotland. Due to subject anonymity, characteristics such as schizophrenia- status, age and ethnic group could not be determined. However at the time of sample collection, the Aberdeen Blood Transfusion Service made a concerted effort to exclude any non-Caucasian samples. At the time of this study, nonCaucasians constituted around $2 \%$ of the Aberdeen population but a lower percentage of Aberdeen blood donors. 724 control subjects were male; 618 were female. Informed consent was obtained for all subjects involved in this study.

As calculated by the power equations of Risch and Teng [13], under a multiplicative model of association, a sample of 400 cases and 1300 controls has $80 \%$ power to detect disease association at an $\alpha=0.05$ significance level for SNPs having a genotypic relative risk of 1.5 [14] and susceptibility allele frequencies between 0.07 and 0.9 .

\section{Polymorphism selection and genotyping}

SNPs from each gene were selected at an approximate spacing of 1 SNP every $10 \mathrm{~kb}$. Six of these SNPs are located in potentially functional regions of the genes, five in exons and one in a promoter region (Table 1). Among the SNPs that are in exons, two are synonymous, but the other three produce amino acid changes. The size of these genes varied greatly, from $23 \mathrm{~kb}$ to $1110 \mathrm{~kb}$, and there was a corresponding variation in the number of SNPs analysed, from 4 to 109 (for further details see Table 2, in the Results section).

Genotyping was performed using a modification of the single base chain extension assay previously described [15], at GlaxoSmithKline, UK. A SNP passed QC if over $80 \%$ of genotype calls were successful.

\section{Statistical analysis}

All SNPs were tested for departure from Hardy Weinberg equilibrium (HWE) in controls using a chi squared test with one degree of freedom. This test compares the expected genotype frequencies calculated from the allele frequencies, with observed genotype frequencies. A departure from HWE in controls may indicate errors in the data.

Association testing of both alleles and genotypes was carried out using the 'PROC FREQ' fast Fisher's Exact Test (FET) procedure in the statistical software package SASv8.2 (SAS Institute Inc., Cary, NC, USA). The Fast Fisher's Exact Test computes exact $P$-values for general contingency tables using the network algorithm developed by [16]. The network algorithm provides substantial advantages over direct enumeration and is rapid and accurate. The exact test is used in preference to the asymptotic chi-square $P$-value as it is more accurate at loci where the minor allele frequency is low.

In order to model and test for interactions between SNPs in different candidate genes, standard logistic regression analysis was performed using the statistical software package R (The R Project for Statistical Computing, [17]). Following Cordell [18] the model tested had the following form:

$$
\begin{gathered}
\log (p /(1-p))=\mu+a_{1} x_{1}+d_{1} z_{1}+a_{2} x_{2}+d_{2} z_{2}+i_{a a} x_{1} x_{2}+i_{a d} x_{1} \\
z_{2}+i_{d a} z_{1} x_{2}+i_{d d} z_{1} z_{2}
\end{gathered}
$$

where $x_{1}, x_{2}, z_{1}$ and $z_{2}$ are variables determined by the genotype under consideration, and $\mu, a_{1}, a_{2}, d_{1}, d_{2}, i_{a a^{\prime}} i_{a d^{\prime}} i_{d a}$ and $i_{d d}$ are parameters to be estimated. Here, genotypes at two SNPs are being modelled and, for each of the 9 possible genotype combinations at the two SNPs, $p$ is the proportion of subjects with the genotype who are cases and $(1-p)$ is the proportion of subjects with the genotype who are controls. The first five terms in the equation represent the effects of the two SNPs on $\log (p /(1-p))$ if there is no statistical interactions between the two SNPs. A non-zero value for one or more of the remaining four coefficients $\left(i_{a a^{\prime}}, i_{a d^{\prime}} i_{d a^{\prime}} i_{d d}\right)$ indicates the presence of interaction. Statis-

\begin{tabular}{|c|c|c|c|c|c|c|}
\hline Gene & Polymorphism ' & Chromosome & Position ${ }^{2}$ & Region & Base change & $\begin{array}{l}\text { Amino acid } \\
\text { change }\end{array}$ \\
\hline EGFR & rs2072454 & 7 & 54988557 & Exon 4 & $\mathrm{C} / \mathrm{T}$ & Asn/Asn \\
\hline ERBB2 & rs 1801200 & 17 & 35133114 & Exon 20 & $A / G$ & $\mathrm{Val} / \mathrm{lle}$ \\
\hline ERBB2 & rs 1058808 & 17 & 35137563 & Exon 30 & $\mathrm{C} / \mathrm{G}$ & Pro/Ala \\
\hline ERBB4 & rs3748962 & 2 & 212077370 & Exon 27 & $\mathrm{C} / \mathrm{T}$ & $\mathrm{Val} / \mathrm{Val}$ \\
\hline NRGI & rs6994992 & 8 & 31615123 & Promoter & $\mathrm{C} / \mathrm{T}$ & - \\
\hline NRGI & rs3924999 & 8 & 32572900 & Exon 2 & $\mathrm{C} / \mathrm{T}$ & Gln/Arg \\
\hline
\end{tabular}

Table I: SNPs in potentially functional gene regions included in the present study.

'Polymorphism names are taken from the dbSNP website, [47].

${ }^{2}$ From map NCBI 35. 
Table 2: Polymorphism in genes in the families NRG and ErbB investigated in this study.

\begin{tabular}{|c|c|c|c|c|c|}
\hline Gene & Chromosome & Size (kb) & No. of analysable SNPs & No. of SNPs with $p<0.101$ & No. of SNPs with $p<0.05$ \\
\hline NRGI & 8 & 1120 & 94 & $22(23 \%)$ & $11(12 \%)$ \\
\hline NRG2 & 5 & 196 & 16 & $2(13 \%)$ & I (6\%) \\
\hline NRG3 & 10 & 1110 & 105 & $21(20 \%)$ & $10(10 \%)$ \\
\hline NRG4 & 15 & 70 & 9 & 0 & 0 \\
\hline EGFR & 7 & 188 & 22 & $7(32 \%)$ & $6(27 \%)$ \\
\hline ERBB2 & 17 & 29 & 4 & I (25\%) & 0 \\
\hline ERBB3 & 12 & 23 & 6 & 0 & 0 \\
\hline ERBB4 & 2 & 1160 & 109 & $26(24 \%)$ & 14 (I3\%) \\
\hline
\end{tabular}

I Either genotypic test or allelic test of association.

tical significance was assessed by a likelihood ratio test, comparing the full model above with a reduced model in which the last four terms are absent (i.e. in which the coefficients $i_{a a^{\prime}} i_{a d^{\prime}} i_{d a}$ and $i_{d d}$ are all set to zero).

A significant interaction effect in the logistic regression analysis indicates that the combined effect of the two SNPs is significantly different from the sum of their individual effects. Pair-wise interactions may be of three types, namely 'and', 'or' and 'exclusive or', defined as follows. In an 'and' relationship, the effect of having the risk-enhancing genotypes at both SNP loci is larger than the sum of the single-locus effects. In an 'or' relationship, the effect of having the risk-enhancing genotype at both loci is smaller than the sum of the single-locus effects. In the final type of interaction, named 'exclusive or', the effect of having the risk-enhancing genotype at both loci is smaller than that of having a risk-enhancing genotype at only one locus. For each SNP pair, each of these configurations gives rise to a contingency table, classified by genotype group (risk-enhancing or risk-reducing) and phenotype (case or control). These contingency tables were evaluated, and the configuration with the lowest $P$-value for association with the phenotype was identified. This configuration indicated the type of interaction between the two loci in question.

\section{Results \\ Data checking}

Although controls were selected from the same geographic area as the cases, a test was conducted to determine the presence of any latent population stratification. The lambda statistic of Devlin et al. [19], calculated on 4,250 SNPs distributed over the whole genome, showed no evidence of stratification (population heterogeneity, overdispersion: Genotypic exact lambda $=1.061$, Allelic exact lambda $=1.000)$, indicating that no genomic-control adjustment is required when performing tests of genotypic and allelic association on these subjects.
Of 374 SNPs submitted for genotyping, 3 were dropped from analysis due to monomorphism in cases and controls, and another 6 were dropped due to departure from Hardy-Weinberg Equilibrium $(P<0.001)$. Among the remaining SNPs, the proportion of individuals successfully genotyped ranged from 0.804 to 0.99 , with a median of 0.97. A subset of the individuals studied was genotyped in duplicate, giving a total of 14,323 duplicate genotypes. Of these, only $2(=0.014 \%)$ were inconsistent. The 365 SNPs retained, located in 8 genes, were subjected to single-point analysis. The number of SNPs per gene is given in Table 2. The median frequency of the minor allele among the SNPs analysed was $22.9 \%$, but there were 35 SNPs (9.6\% of those analysed) for which the minor allele frequency was less than $5 \%$. These SNPs, selected prior to the availability of the HapMap [20], are less informative than the others; however, as they do provide some additional information they were retained in the analysis.

\section{Single-point results}

Both allelic and genotypic tests of association were conducted. Detailed results are provided in Additional file 1. Significant results $(P<0.05)$ were observed in five genes, namely NRG1, NRG2, NRG3, EGFR and ERBB4. These confirmed previous findings on NRG1 and ERBB4 and suggest possible involvement also of NRG2, NRG3 and EGFR. Borderline evidence of association $(P<0.10)$ was observed for one SNP in ERBB2. Haplotypic tests of association (not shown) provided similar results to the singlepoint results described here. If none of the 365 single SNPs were associated with the disease status (the null hypothesis), we would expect 18 SNPs to be significant at the 0.05 level and 37 SNPs to be significant at the 0.10 level on an allelic test. For the allelic test there were 34 SNPs significant at the 0.05 level (9.3\%) and 59 SNPs significant at the 0.10 level $(16.2 \%)$. For the genotypic test there were 24 SNPs significant at the 0.05 level (6.6\%) and 62 SNPs significant at the 0.10 level $(17.0 \%)$. This excess of significantly associated SNPs, beyond the num- 
bers expected by chance when accounting for the multiple tests, provides evidence that some of these associations are real.

The distribution of evidence for single-point association with schizophrenia, and linkage disequilibrium (LD), over gene NRG1 are presented in Fig. 2. In this figure, the magnitude of association of each SNP with schizophrenia is indicated by the log-transformed $P$-value. For the sake of simplicity, only values arising from the genotypic exact test are shown; allelic test results followed a very similar pattern. Across both tests, eleven SNPs in this gene showed significant genotypic association with schizophrenia $(P<0.05)$, and in three instances the association was highly significant $(P<0.01)$ (see Additional file 1 : SNP RS776401: map position 031836504 on Chr 8, risk genotype odds ratio $(\mathrm{OR})=1.461,95 \%$ confidence interval (CI) 1.160-1.840; SNP RS1383887: map position 031872698, OR $=1.589$, CI 1.213-2.081; SNP RSGSK8116812: map position 032741837, OR $=1.651$, CI 1.242-2.196). A putatively functional SNP in Exon 2, coding a non-synonymous (Gly/Arg) change, showed allelic association to schizophrenia with $P=0.0279$ (SNP RS3924999: map position 032572900, OR $=1.302$, CI 1.028-1.648). It is possible that the cluster of associations observed at the 3' end of the gene, to the right of the figure, has arisen through LD with this non-synonymous change or another underlying functional variant. Further evidence of association is seen at the $5^{\prime}$ end of the gene, closer to Exon 1. The latter associations fall beyond the apparent reach of $\mathrm{LD}$.

The corresponding information for gene ERBB4 is presented in Fig. 3. In this gene, fourteen SNPs were significantly associated with schizophrenia by either the allelic or genotypic test, including one allelic test result which was highly significant $(P<0.01$ : SNP RS6754674: map position 213033762 on Chr 2, OR = 1.387, CI 1.0561.822). Again, the graph of genotypic test results shows strong evidence of association occurring most commonly at the two ends of the gene, particularly at the 5 '-end, to the right of the figure, although the pattern is not as clear as in the case of NRG1. This region of the gene represents a $0.15 \mathrm{Mb}$ region of moderate $\mathrm{LD}$, so it is possible that a single underlying variant, in LD with all of the SNPs that show significant association, accounts for the observed association in this region.

The corresponding information for genes NRG2, NRG3, EGFR and ERBB2 is presented in Fig. 4, except that the LD maps are omitted for brevity. A single SNP in NRG2 showed significant genotypic association with schizophrenia (SNP RS2936651: map position 139401129 on Chr 5, OR = 2.081, CI 1.156-3.745). This finding was corroborated by a significant allelic test result also. Ten SNPs were significantly associated in NRG3, including one which was highly significantly associated $(P<0.01)$ by the allelic test (RS3924461: map position 084423371 on Chr10, OR = 1.283, CI 1.077-1.529). The pattern of LD in this gene (results not shown) is highly localised, similar to that in NRG1, so the observation of association from spatially separated SNPs may have arisen by chance or may suggest the presence of different functional variants within the same gene. The spatial pattern of association is not related to the distribution of the exons in this gene. Six SNPs in EGFR were significantly associated with schizophrenia, however, all fell in the range $0.01<P<0.05$. Only four SNPs were studied in ERBB2, and none of these was found to be significantly associated with schizophrenia.

\section{Interaction analysis results}

As summarised in Table 2, 79 SNPs were identified which had a $P$-value from an allelic or genotypic test that was less than 0.10 (details are given in Additional file 2). These SNPs were contained in the 6 genes considered above, namely NRG1, NRG2, NRG3, EGFR, ERBB2, and ERBB4. All pairs of these SNPs that are at least $0.5 \mathrm{cM}$ apart on an interpolated Decode genetic map [21] were tested for interaction effects in a logistic regression interaction analysis.

A total of 2640 statistical tests for pair-wise interactions were performed (details of results are given in Additional file 2). Of these, 212 were significant at the $5 \%$ level: that is $8.03 \%$ of all tests were significant compared to 132 (= $5 \%$ of 2640) expected by chance if the null hypothesis of no interaction were true for all pairs of SNPs tested. If all the tests performed were mutually independent the probability that 212 or more would be significant by chance is $2.575 \times 10^{-11}(\mathrm{P}(R \geq 212)$ where $R \sim \operatorname{binomial}(N=2640, p$ $=0.05)$ ). The true probability of such an outcome is certain to be larger, due to non-independence among the tests: nevertheless, this result provides convincing evidence that some of the observed interactions are real. The significance levels presented here are not adjusted for multiple testing: a Bonferroni correction would be inappropriate due to non-independence among the tests, and the multiple-testing issue is addressed by the comparison of observed and expected numbers of significant tests presented above. None of the significant results obtained would survive the Bonferroni correction.

Table 3 gives the percentage of significant interactions for each gene combination; the corresponding counts are given in brackets. There are four gene combinations in which the percentage of significant interactions is substantially higher than the $5 \%$ expected by chance, viz. the two-gene combinations NRG1-NRG2, NRG1-NRG3 and NRG2-EGFR, and the single large gene ERBB4. The percentages of significant interactions for combinations 

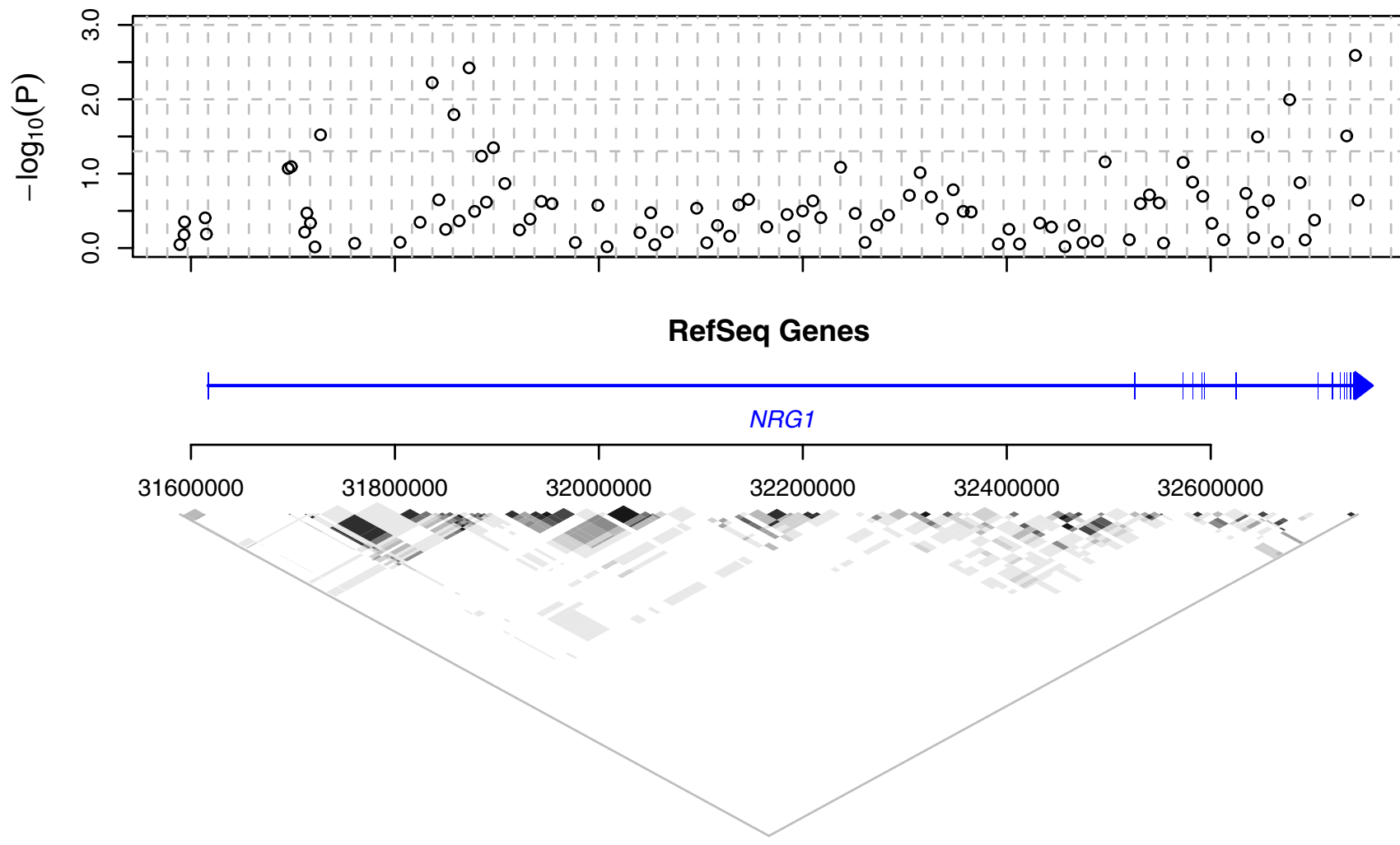

Chromosome Position (bp)

Figure 2

Genotypic single-point association test results and LD over NRG I. In the plot of - $\log _{10}(P)$ v. map position, horizontal dashed lines are at $P=0.05,0.0 \mathrm{I}$ and $0.00 \mathrm{I}$. Vertical dashed lines are at $20 \mathrm{~kb}$ intervals. The region covered by the gene is indicated in the centre of the figure by a horizontal line, with an arrow head indicating the direction of transcription. A crossing vertical line indicates the position of each exon. At the base of the figure, LD between SNP loci is indicated by shading. White: $r^{2}=0$; black: $r^{2}=1$.

NRG1-NRG2 and NRG2-EGFR are based on a small number of tests and should therefore be treated with caution, but the percentages for NRG1-NRG3 and ERBB4 are based on substantial numbers.

The next step towards the identification of a mechanism to account for the statistical interactions is the identification of the type of each significant interaction. This was performed by pair-wise combination analysis (see Methods). Fig. 5 illustrates the distribution of the three types of interaction over the four gene combinations with a large percentage of significant interactions. The frequencies of and-type and or-type interactions are fairly similar. This might be expected, as the patterns of results that correspond to these two types of interaction are complementary, and equally likely to occur by chance. Interactions of the xor-type are rare: indeed, they are observed only in the two gene combinations that gave rise to a fairly large sam- ple of significant interactions. This also might be expected, as it corresponds to a complex biological mechanism and to a rather specific pattern of results that is relatively unlikely to occur by chance.

\section{Discussion}

In this manuscript we present confirmation of earlier published associations between schizophrenia and the genes NRG1 and ERBB4. We build a new, compelling picture of pathway involvement by showing that associations exist with additional gene family-members, namely EGFR, NRG2, and NRG3. Lastly, we present statistical evidence of gene epistasis, consistent with a highly complex biological pathway of schizophrenia susceptibility. This is the first time that the roles and inter-relationships of the ERBB and NRG gene families as a whole have been investigated in relation to schizophrenia and our approach sets a precedent in the investigation of common complex diseases. 

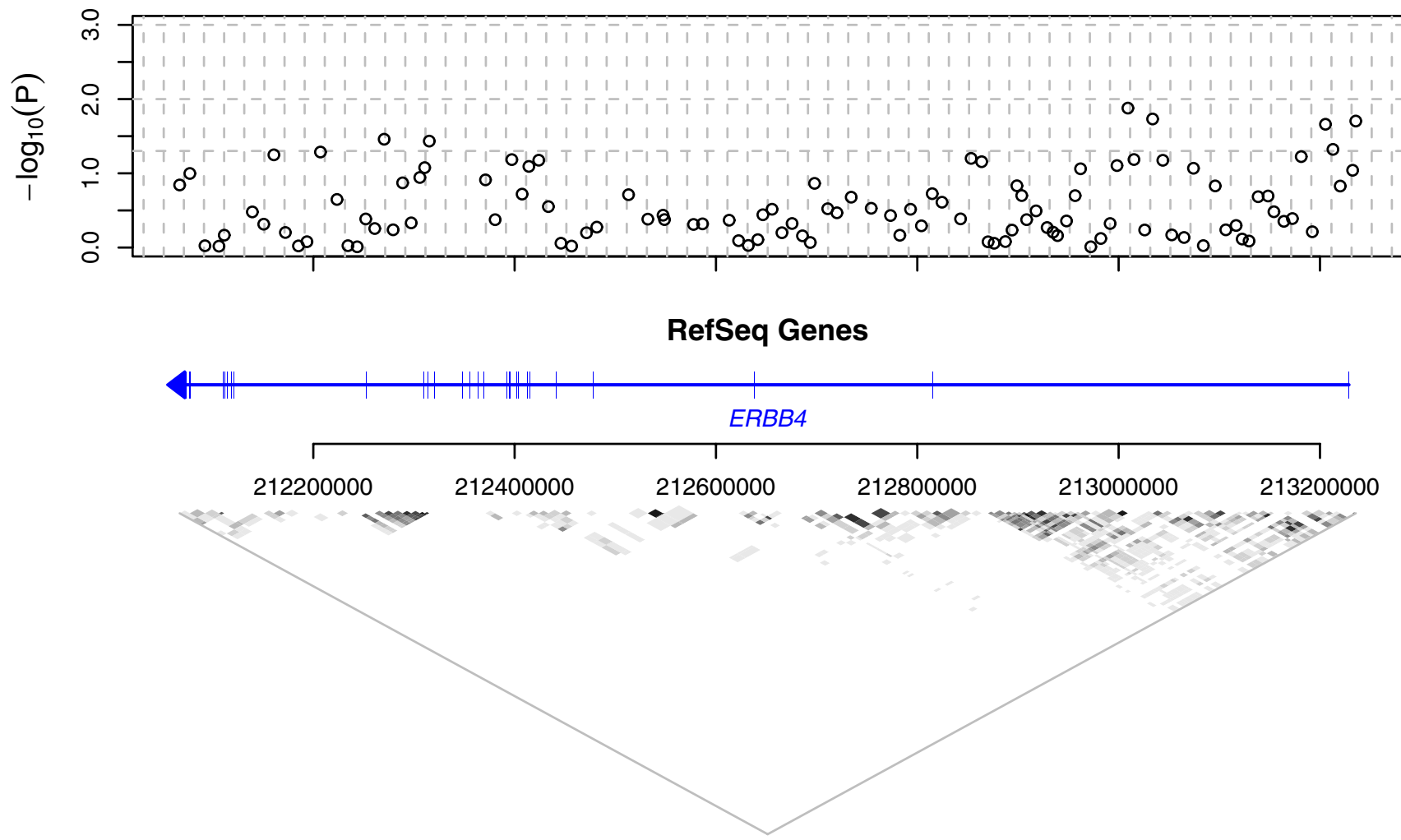

Chromosome Position (bp)

Figure 3

Genotypic single-point association test results and LD over ERBB4. In the plot of - $\log _{10}(P)$ v. map position, horizontal dashed lines are at $P=0.05,0.0 \mathrm{I}$ and $0.00 \mathrm{I}$. Vertical dashed lines are at $20 \mathrm{~kb}$ intervals. The region covered by the gene is indicated in the centre of the figure by a horizontal line, with an arrow head indicating the direction of transcription. A crossing vertical line indicates the position of each exon. At the base of the figure, LD between SNP loci is indicated by shading. White: $r^{2}=0$; black: $r^{2}=1$.

Clear evidence for gene-gene interaction was detected for gene combinations NRG1-NRG2, NRG1-NRG3 and EGFR-NRG2, and suggestive evidence was also seen for ERBB4-NRG1, ERBB4-NRG2, ERBB4-NRG3 and ERBB4ERBB2. Evidence of intragenic interaction was seen for SNPs in ERBB4. Of the 2640 tests for pair-wise interaction, 212 were significant at the $5 \%$ level, which is substantially more than the 131 expected by chance. This large number of low $P$-values may be partly explained by non-independence among tests. However, it may also indicate that some of the 79 SNPs studied interact to modulate susceptibility to schizophrenia.

Numerous studies have investigated the association between schizophrenia and NRG1 (reviewed in [22]). A comparison of the associations generated in the current study with those previously published shows good agreement. Associations that we observed at the 5 ' region of
NRG1 overlap with those found by Stefansson et al. $[3,4]$, Corvin et al. [23], Li et al. [24], Zhao et al. [25] and Petryshen et al. [26]. Similarly, the associations we observed at the 3 ' end of the gene overlap with those found by Yang et al. [27], Li et al. [23], Petryshen et al. [25] and Lachmann et al. [28]. Two polymorphisms in our study directly replicate these associations: the putatively functional SNP rs3924999 was found to be associated with schizophrenia in a Han Chinese family study conducted by Yang et al. [26]. This SNP was also tested in a Caucasian study of 136 families by Duan et al. [29], but was not significant. Rs2466049 was significant as part of a haplotype found in a Caucasian family by Petryshen et al. [25].

For the ERBB4 gene, four polymorphisms (rs3748962, rs839523, rs2054617 and rs6435711) screened in this study were also analysed by Silberberg et al. [8]. However, none of the individual SNP associations observed in the 
a) NRG2
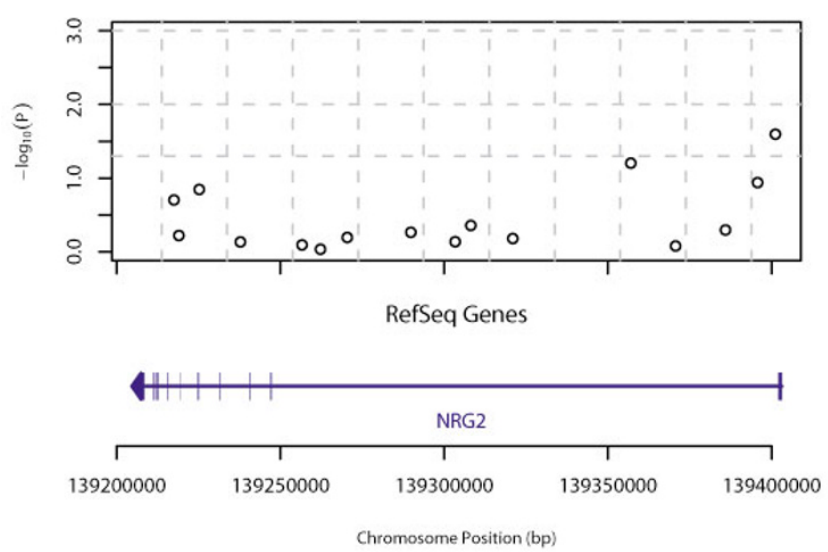

c) EGFR

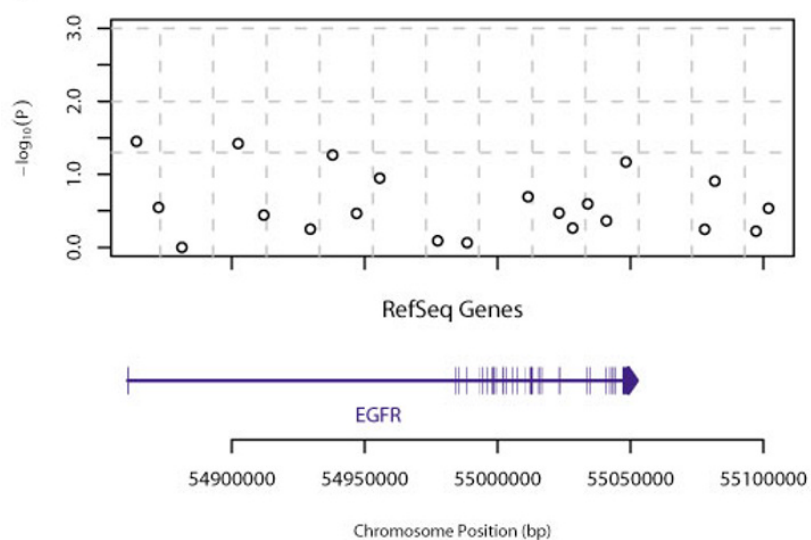

b) NRG3
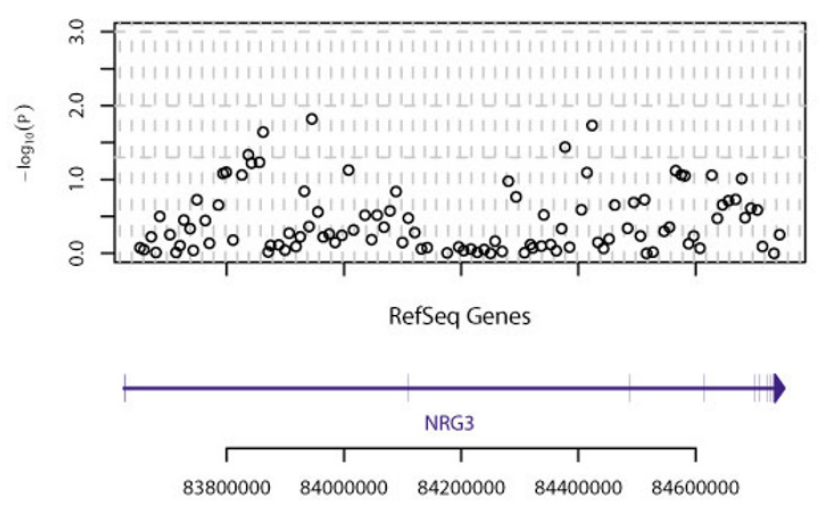

Chromosome Position (bp)

d) ERBB2

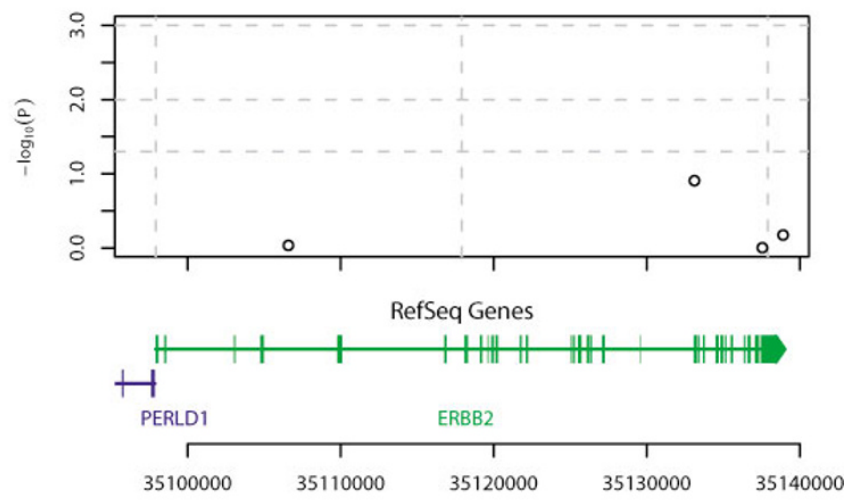

Chromosome Position (bp)

Figure 4

Genotypic single-point association test results and LD over NRG2, NRG3, EGFR and ERBB2. In the plot of $\log _{10}(P)$ v. map position, horizontal dashed lines are at $P=0.05,0.0 \mathrm{I}$ and $0.00 \mathrm{I}$. Vertical dashed lines are at $20 \mathrm{~kb}$ intervals. The region covered by the gene is indicated in the centre of the figure by a horizontal line, with an arrow head indicating the direction of transcription. A crossing vertical line indicates the position of each exon.

previous study was replicated here. As no underlying functional variant has been identified for ERBB4, this lack of association at the SNP level is unsurprising and is consistent with a heterogeneous haplotypic background across studies. It is worth noting also that non-coding polymorphisms might lead to dysregulation of ErbB4 expression levels, a hypothesis corroborated by the Silberberg et al. [8] study, which found enhanced expression of the ERBB4 Jm-a CYT-1 isoform (one of four splice variants, see [30]) in schizophrenic patients. The altered balance of ErbB4 isoforms could lead to variations in homo- and heterodimer formation and thus in downstream signalling pathways.

In addition to providing further evidence of NRG1 and ERBB4 associations to schizophrenia, our study is the first to suggest possible involvement of three additional genes from the NRG and ERBB gene families, i.e. NRG2, NRG3 and EGFR (ERBB1). It is interesting to note that we only detected evidence for association of NRG genes which are expressed in the central nervous system, namely NRG1, NRG2 and the nervous-system-specific NRG3 [31], whereas NRG4, which is mainly expressed in pancreas and absent from the brain [32] was not found to be associated to schizophrenia.

To our knowledge, an association of EGFR to schizophrenia has not previously been investigated. Association of a polymorphism in EGF (one of the six EGFR ligands, Fig. 1) with schizophrenia was reported in Finnish men [33], but not replicated in a Japanese population [34]. Further support for a possible role of EGF and EGFR in the disease comes from findings of abnormal expression of these genes in the forebrain and serum of schizophrenic 
Table 3: Percentages of pair-wise interactions that were significant $(P<0.05)$, classified by the gene(s) in which the interacting SNPs lie. Gene combinations in which there are substantially more significant interactions than the $5 \%$ expected by chance are indicated in bold. The counts that contribute to each percentage are also shown in parentheses as a fraction. The presence of "-" in the table indicates that no pairs of SNPs in this gene are $0.5 \mathrm{cM}$ apart.

\begin{tabular}{|c|c|c|c|c|c|c|}
\hline & NRG I & NRG2 & NRG3 & EGFR & ERBB2 & ERBB4 \\
\hline NRG I & $5.8 \%(6 / 103)$ & $13.6 \%(6 / 44)$ & | I.7\% (54/462) & $4.5 \%(7 / 154)$ & $0.0 \%(0 / 22)$ & $7.9 \%(45 / 572)$ \\
\hline NRG2 & & - & $4.8 \%(2 / 42)$ & $14.3 \%(2 / 14)$ & $0.0 \%(0 / 2)$ & $7.7 \%(4 / 52)$ \\
\hline NRG3 & & & $5.7 \%(6 / 105)$ & $2.0 \%(3 / 147)$ & $0.0 \%(0 / 21)$ & $8.4 \%(46 / 546)$ \\
\hline EGFR & & & & - & $0.0 \%(0 / 7)$ & $5.5 \%(10 / 182)$ \\
\hline ERBB2 & & & & & - & $7.7 \%(2 / 26)$ \\
\hline ERBB4 & & & & & & $13.7 \%(19 / 139)$ \\
\hline
\end{tabular}

patients [35]. EGFR is usually omitted in reviews on NRGERBB signalling, although it is well-established that ErbB4 can interact with and cross-phosphorylate EGFR upon neuregulin-binding [36-39]. Furthermore, EGFR and ERBB4 show partially overlapping temporal and spatial mRNA expression in several brain areas, such as cortex, striatum, cerebellum and hippocampus, and are coexpressed in GABAergic interneurons [40,41]. Therefore, it is very likely that EGFR/ErbB4 heterodimers mediate important functions in the developing and adult central nervous system in vivo. Whilst we limited this study to the NRG and ERBB gene families, it would be interesting to investigate association of the additional EGFR and ErbB4 ligands (see Fig. 1).

The significant gene-gene interactions reported in this study support our hypothesis of pathway involvement in schizophrenia. These genes have related functions and, for example, the NRG1-NRG2 and NRG1-NRG3 interactions may suggest competition between these ligands for a

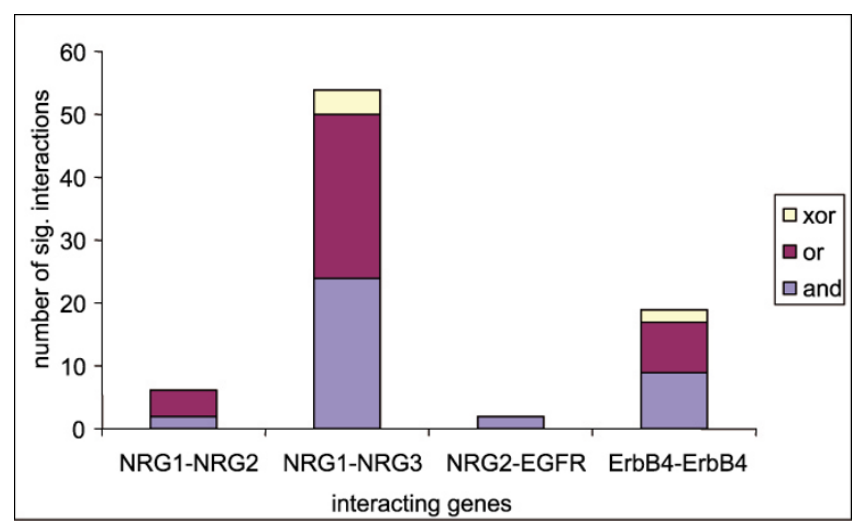

\section{Figure 5}

Distribution of the types of pair-wise interaction in gene combinations with a large percentage of significant interactions. The total in each column corresponds to the number of significant interactions presented in Table 3 , i.e. the numerator of the fraction presented in that table for the gene combination in question. receptor. The NRG2-EGFR interaction is superficially more difficult to explain since NRG2 is a ligand for ErbB3 and ErbB4 and does not usually recognize EGFR directly or with high affinity [42-44]. However, a recent study reports that a single amino acid change in the EGFR ligand-binding domain dramatically increases the receptor affinity for NRG $2 \beta$ which can then induce potent stimulation of EGFR signalling [45]. The interactions within the ERBB4 gene suggest that combinations of particular genotypes may result in modified receptor activity, leading to altered risk of schizophrenia.

Such suggestions concerning possible mechanisms of interaction remain speculative until the reality of these interactions has been established biologically and the effect of each two-locus genotype on the phenotype is understood. A follow-up study currently underway, using independent cases and controls, will clarify which of these interactions can be replicated and may generate further hypotheses regarding the nature of the interactions. It will be interesting also to investigate whether observed associations or their interactions correspond to particular phenotypically defined patient sub-groups. The strong evidence of genetic associations and interactions reported here provides ample justification for such an enquiry.

\section{Conclusion}

This study confirms previously published associations between schizophrenia and NRG1 and ERBB4, and points to three other members of the NRG and ERBB gene families (EGFR, NRG2 and NRG3) as potential schizophrenia susceptibility genes. Furthermore, the study presents evidence for inter- and intragenic pair-wise interaction between loci in genes of both families (NRG1-NRG2, NRG2-EGFR, NRG1-NRG3 and ERBB4-ERBB4). These extensive genetic inter-relationships observed among ligands and receptors of the ERBB and NRG gene families reflect the complexity of their interactions at the molecular level. The influence of so many closely related genetic factors on the potential development of disease pathophysiology has rarely, if ever, been demonstrated before and may partially explain the difficulty in arriving at an 
overarching genetic hypothesis of the etiology of schizophrenia. In future studies, it will be important to replicate our new findings in an independent cohort and to understand the underlying biological mechanisms of the singlelocus associations as well as the interactions among different loci.

\section{Competing interests}

The author(s) declare that they have no competing interests.

\section{Authors' contributions}

IB helped to formulate the study and identified the genes of interest; she led the writing of the manuscript and provided the biological interpretation and context to our results. $\mathrm{AB}$ led the statistical analysis, and produced the first draft of the manuscript. BB performed the singlepoint statistical analyses and the pairwise analysis, and provided interpretation of his results. NG provided interpretation of the linkage disequilibrium analysis and substantially contributed to the interpretation of the statistical analyses in the manuscript. PM was instrumental in progressing the study, revised the manuscript and added important intellectual content concerning the biology of schizophrenia. RM designed the statistical analysis plan and provided schizophrenia genetics expertise to the writing of the manuscript. DS designed the laboratory aspects of the experiment and oversaw their execution. DSC recruited, worked up clinically and supplied DNA from the Aberdeen schizophrenia case collection of 396 cases. PY provided the control collection of 1342 anonymous blood donors from Aberdeen. IP helped to formulate the original idea for the study and revised the manuscript critically, providing important intellectual content. All authors read, edited and approved the final manuscript.

\section{Additional material}

\section{Additional file 1}

Detailed results from allelic and genotypic tests of single-point association of SNP loci with schizophrenia.

Click here for file

[http://www.biomedcentral.com/content/supplementary/17449081-3-31-S1.xls]

\section{Additional file 2}

Detailed results from allelic and genotypic tests of association between SNP-pairs and susceptibility to schizophrenia.

Click here for file

[http://www.biomedcentral.com/content/supplementary/17449081-3-31-S2.xls]

\section{Acknowledgements}

The authors wish to acknowledge the patients and blood donors who contributed to this study. Thanks are also due to members of the GSK Genetic Sample Management and Genotyping groups; also Matt Nelson, Pierandrea Muglia, Paul Skelding and Nicola Foote for their helpful contributions in the writing of this paper.

\section{References}

I. Owen MJ, Craddock N, O'Donovan MC: Schizophrenia: genes at last? [Review]. Trends in Genetics 2005, $21: 5$ | 8-525.

2. Harrison PJ, Weinberger DR: Schizophrenia genes, gene expression, and neuropathology: on the matter of their convergence [Review]. Molecular Psychiatry 2005, 10:40-68.

3. Stefansson H, Sigurdsson E, Steinthorsdottir V, Bjornsdottir S, Sigmundsson T, Ghosh S, Brynjolfsson J, Gunnarsdottir S, Ivarsson O, Chou TT, Hjaltason O, Birgisdottir B, Jonsson H, Gudnadottir VG, Gudmundsdottir E, Bjornsson A, Ingvarsson B, Ingason A, Sigfusson S, Hardardottir H, Harvey RP, Lai D, Zhou M, Brunner D, Mutel V, Gonzalo A, Lemke G, Sainz J, Johannesson G, Andresson T, Gudbjartsson D, Manolescu A, Frigge ML, Gurney ME, Kong A, Gulcher JR, Petursson $\mathrm{H}$, Stefansson K: Neuregulin I and susceptibility to schizophrenia. Am J Hum Genet 2002, 7 I:877-892.

4. Stefansson H, Sarginson J, Kong A, Yates P, Steinthorsdottir V, Gudfinnsson E, Gunnarsdottir S, Walker N, Petursson H, Crombie C, Ingason A, Gulcher JR, Stefansson K, St Clair D: Association of neuregulin I with schizophrenia confirmed in a Scottish population. Am J Hum Genet 2003, 72:83-87.

5. Tosato S, Dazzan P, Collier D: Association between the neuregulin I gene and schizophrenia: a systematic review. Schizophr Bull 2005, 31:613-617.

6. Harrison PJ, Law AJ: Neuregulin I and Schizophrenia: Genetics, Gene Expression, and Neurobiology. Biol Psychiatry 2006, 60:132-140.

7. Norton N, Moskvina V, Morris DW, Bray NJ, Zammit S, Williams NM, Williams HJ, Preece AC, Dwyer S, Wilkinson JC, Spurlock G, Kirov G, Buckland P, Waddington JL, Gill M, Corvin AP, Owen MJ, O'Donovan MC: Evidence that interaction between neuregulin $I$ and its receptor erbB4 increases susceptibility to schizophrenia. Am J Med Genet B Neuropsychiatr Genet 2006, I 41:96-101.

8. Silberberg G, Darvasi A, Pinkas-Kramarski R, Navon R: The involvement of ErbB4 with schizophrenia: association and expression studies. Am J Med Genet B Neuropsychiatr Genet 2006, |41:142-148.

9. Nicodemus KK, Luna A, Vakkalanka R, Goldberg T, Egan M, Straub RE, Weinberger DR: Further evidence for association between ErbB4 and schizophrenia and influence on cognitive intermediate phenotypes in healthy controls. Molecular Psychiatry 2006, II:1062-1065.

10. Falls DL: Neuregulins: functions, forms, and signaling strategies. Exp Cell Res 2003, 284:14-30.

II. Talmage DA, Role LW: Multiple personalities of neuregulin gene family members. J Comp Neurol 2004, 472: I34- 139.

12. Yarden Y, Sliwkowski MX: Untangling the ErbB signalling network. Nat Rev Mol Cell Biol 200I, 2:127-137.

13. Risch N, Teng J: The relative power of family-based and casecontrol designs for linkage disequilibrium studies of complex human diseases - I. DNA pooling. Genome Research 1998, 8: $1273-1288$.

14. Risch N, Merikangas $\mathrm{K}$ : The future of genetic studies of complex human diseases. Science 1996, 273:1516-1517.

15. Taylor JD, Briley D, Nguyen Q, Long K, lannone MA, Li MS, Ye F, Afshari A, Lai E, Wagner M, Chen J, Weiner MP: Flow cytometric platform for high-throughput single nucleotide polymorphism analysis. Biotechniques 2001, 30:661-669.

16. CR M, NR P: A network algorithm for performing Fisher's Exact Test in $\mathbf{r} \times \mathbf{c}$ contingency tables. J Am Stat Assoc 1983, 78:427-434.

17. The R Project for Statistical Computing 2003 [http://www.rproject.org/].

18. Cordell H]: Epistasis: what it means, what it doesn't mean, and statistical methods to detect it in humans. Hum Mol Genet 2002, I I:2463-2468. 
19. Devlin B, Roeder K, Wasserman L: Genomic control, a new approach to genetic-based association studies. Theoretical Population Biology 2001, 60:155-166.

20. Altshuler D, Brooks LD, Chakravarti A, Collins FS, Daly MJ, Donnelly P: A haplotype map of the human genome. Nature 2005, 437: $1299-1320$.

21. Kong A, Gudbjartsson DF, Sainz J, Jonsdottir GM, Gudjonsson SA Richardsson B, Sigurdardottir S, Barnard J, Hallbeck B, Masson G, Shlien A, Palsson ST, Frigge ML, Thorgeirsson TE, Gulcher JR, Stefansson $\mathrm{K}$ : A high-resolution recombination map of the human genome. Nat Genet 2002, 3 1:241-247.

22. Munafo MR, Thiselton DL, Clark TG, Flint J: Association of the NRG I gene and schizophrenia: a meta-analysis. Mol Psychiatry 2006, I I:613.

23. Corvin AP, Morris DW, McGhee K, Schwaiger S, Scully P, Quinn J, Meagher D, Clair DS, Waddington JL, Gill M: Confirmation and refinement of an 'at-risk' haplotype for schizophrenia suggests the EST cluster, Hs.97362, as a potential susceptibility gene at the Neuregulin- I locus. Mol Psychiatry 2004, 9:208-2 I3.

24. Li T, Stefansson H, Gudfinnsson E, Cai G, Liu X, Murray RM, Steinthorsdottir V, Januel D, Gudnadottir VG, Petursson H, Ingason A, Gulcher JR, Stefansson K, Collier DA: Identification of a novel neuregulin I at-risk haplotype in Han schizophrenia Chinese patients, but no association with the Icelandic/Scottish risk haplotype. Mol Psychiatry 2004, 9:698-704.

25. Zhao X, Shi Y, Tang J, Tang R, Yu L, Gu N, Feng G, Zhu S, Liu H, Xing $Y$, Zhao S, Sang H, Guan Y, St Clair D, He L: A case control and family based association study of the neuregulin I gene and schizophrenia. J Med Genet 2004, 4I:31-34.

26. Petryshen TL, Middleton FA, Kirby A, Aldinger KA, Purcell S, Tahl AR, Morley CP, McGann L, Gentile KL, Rockwell GN, Medeiros HM, Carvalho C, Macedo A, Dourado A, Valente J, Ferreira CP, Patterson NJ, Azevedo MH, Daly MJ, Pato CN, Pato MT, Sklar P: Support for involvement of neuregulin I in schizophrenia pathophysiology. Mol Psychiatry 2005, I 0:366-74, 328.

27. Yang JZ, Si TM, Ruan Y, Ling YS, Han YH, Wang XL, Zhou M, Zhang HY, Kong QM, Liu C, Zhang DR, Yu YQ, Liu SZ, Ju GZ, Shu L, Ma DL, Zhang D: Association study of neuregulin I gene with schizophrenia. Mol Psychiatry 2003, 8:706-709.

28. Lachman HM, Pedrosa E, Nolan KA, Glass M, Ye K, Saito T: Analysis of polymorphisms in AT-rich domains of neuregulin I gene in schizophrenia. Am J Med Genet B Neuropsychiatr Genet 2006, | 4 1:102-109.

29. Duan J, Martinez M, Sanders AR, Hou C, Krasner AJ, Schwartz DB, Gejman PV: Neuregulin I (NRG I ) and schizophrenia: analysis of a US family sample and the evidence in the balance. Psychol Med 2005, 35:1599-1610.

30. Junttila TT, Sundvall M, Maatta JA, Elenius K: Erbb4 and its isoforms: selective regulation of growth factor responses by naturally occurring receptor variants. Trends Cardiovasc Med 2000, 10:304-310.

31. Zhang D, Sliwkowski MX, Mark M, Frantz G, Akita R, Sun Y, Hillan K, Crowley C, Brush J, Godowski PJ: Neuregulin-3 (NRG3): a novel neural tissue-enriched protein that binds and activates ErbB4. Proc Natl Acad Sci U S A 1997, 94:9562-9567.

32. Harari D, Tzahar E, Romano J, Shelly M, Pierce JH, Andrews GC Yarden Y: Neuregulin-4: a novel growth factor that acts through the ErbB-4 receptor tyrosine kinase. Oncogene 1999, | 8:268|-2689.

33. Anttila S, Illi A, Kampman O, Mattila KM, Lehtimaki T, Leinonen E: Association of EGF polymorphism with schizophrenia in Finnish men. Neuroreport 2004, I 5:1215-1218.

34. Watanabe Y, Fukui N, Muratake T, Kaneko N, Someya T: No association of EGF polymorphism with schizophrenia in a Japanese population. Neuroreport 2005, 16:403-405.

35. Futamura T, Toyooka K, Iritani S, Niizato K, Nakamura R, Tsuchiya K, Someya T, Kakita A, Takahashi H, Nawa $\mathrm{H}$ : Abnormal expression of epidermal growth factor and its receptor in the forebrain and serum of schizophrenic patients. Mol Psychiatry 2002 7:673-682.

36. Cohen BD, Green JM, Foy L, Fell HP: HER4-mediated biological and biochemical properties in NIH 3 T3 cells. Evidence for HERI-HER4 heterodimers. J Biol Chem 1996, 27 I:48I3-48I8.

37. Hatakeyama M, Yumoto N, Yu X, Shirouzu M, Yokoyama S, Konagaya A: Transformation potency of ErbB heterodimer signaling is determined by B-Raf kinase. Oncogene 2004, 23:5023-503I.
38. Riese DJ, van Raaij TM, Plowman GD, Andrews GC, Stern DF: The cellular response to neuregulins is governed by complex interactions of the erbB receptor family. Mol Cell Biol 1995, I 5:5770-5776.

39. Zhang K, Sun J, Liu N, Wen D, Chang D, Thomason A, Yoshinaga SK: Transformation of NIH 3 T3 cells by HER3 or HER4 receptors requires the presence of HERI or HER2. J Biol Chem I996, 27 I:3884-3890.

40. Fox IJ, Kornblum HI: Developmental profile of ErbB receptors in murine central nervous system: implications for functional interactions. J Neurosci Res 2005, 79:584-597.

41. Kornblum HI, Gall CM, Seroogy KB, Lauterborn JC: A subpopulation of striatal gabaergic neurons expresses the epidermal growth factor receptor. Neuroscience 1995, 69:1025-1029.

42. Carpenter G: ErbB-4: mechanism of action and biology. Exp Cell Res 2003, 284:66-77.

43. Hobbs SS, Coffing SL, Le AT, Cameron EM, Williams EE, Andrew M, Blommel EN, Hammer RP, Chang H, Riese DJ: Neuregulin isoforms exhibit distinct patterns of ErbB family receptor activation. Oncogene 2002, $21: 8442-8452$.

44. Pinkas-Kramarski R, Shelly M, Guarino BC, Wang LM, Lyass L, Alroy I, Alimandi M, Kuo A, Moyer JD, Lavi S, Eisenstein M, Ratzkin BJ, Seger R, Bacus SS, Pierce JH, Andrews GC, Yarden Y: ErbB tyrosine kinases and the two neuregulin families constitute a ligandreceptor network. Mol Cell Biol I998, I 8:6090-6IOI.

45. Gilmore JL, Gallo RM, Riese DJ: The epidermal growth factor receptor (EGFR)-S442F mutant displays increased affinity for neuregulin-2beta and agonist-independent coupling with downstream signalling events. Biochem / 2006, 396:79-88.

46. Olayioye MA, Neve RM, Lane HA, Hynes NE: The ErbB signaling network: receptor heterodimerization in development and cancer. EMBO J 2000, I9:3 | 59-3 I67.

47. NCBI dbSNP website 2004 [http://www.ncbi.nlm.nih.gov/SNP/]
Publish with Biomed Central and every scientist can read your work free of charge

"BioMed Central will be the most significant development for disseminating the results of biomedical research in our lifetime. "

Sir Paul Nurse, Cancer Research UK

Your research papers will be:

- available free of charge to the entire biomedical community

- peer reviewed and published immediately upon acceptance

- cited in PubMed and archived on PubMed Central

- yours - you keep the copyright
BioMedcentral 